\title{
A Novel Model for Train Operation Adjustment in High-Speed Railway based on Max-plus Algebra
}

\author{
Zhong-bo Liu ${ }^{1,2}$ \\ 1. Jilin Communications Polytechnic, Changchun130012, Jilin, China \\ 2. College of Transportation, Jilin University, Changchun 130022, Jilin, China
}

Received: August 17, 2020. Revised: November 27, 2020. Accepted: December 1, 2020. Published: December 2, 2020.

\begin{abstract}
The running time of high-speed train is generally not late, and it can run normally in most cases. However, when severe weather conditions, train components and equipment, accidents or emergencies occur, it will lead to train operation delay and traffic congestion. Therefore, when an accident occurs, we need to adjust the train time or route timely and accurately. As an important algebraic system, max-plus algebra is widely used in the field of industrial production control. In industrial production, the most production mode is the discrete system, but the characteristics and the ability of discrete systems depends on the periodic of system and the number of workpiece produced by the system in unit time, and the characteristics of the system are closely related to the properties of the matrix, especially, the eigenvalues and eigenvectors of the matrix in the sense of max-plus algebra. Therefore, this paper studies the max-plus algebra theory and the solution of eigenvalues and eigenvectors of matrices in the sense of max-plus algebra, establish the operation time matrix to optimize the train operation adjustment model of high-speed railway, and analyze the failure propagation model.
\end{abstract}

Keywords - max-plus algebra, eigenvalue, eigenvector, strategy optimization, failure analysis.

\section{INTRODUCTION}

I $\mathrm{n}$ recent years, industrial and academic circles have begun to study various kinds of discrete event systems (DES), such as flexible manufacturing, remote network, parallel processing, traffic control and so on. The French G.Cohen et al. Proposed the theory method of max algebra in 1985, because of its simple algorithm, it was applied to solve the discrete event dynamic system, It can be used to transform discrete events in ordinary meaning into linear events in max-plus meaning.

The domestic and foreign scholars have also studied it. Fard [1] analysis production scheduling of manufacturing systems by max algebra method, established automatic scheduling mechanism, the process route matrix and equipment part manufacturing time matrix are compatible, through the optimization of simulated annealing algorithm, make it jump out of local optimal solution so as to achieve the global optimal solution. Chen Jin etc.[2]investigates the solvability of maxplus mixed linear inequality systems. By applying the characteristic for the solvability of max-plus linear equation systems and the maximum solution of max-plus mixed linear inequality systems, they present a necessary and sufficient condition for the solvability of max-plus mixed linear inequality systems. Based on the single route model, according to the max algebra theory, Lu Zhiwei[3] designed the terminal convergence route model, and the conflict of aircrafts convergence in the same altitude is solved. It can avoid conflict of intersection point of two linear routes, and a-chieve conflict free 4D trajectory prediction. Zhou X.[4] studied the matrix and vector of the interval coefficients with maximal additive algebras, and gave a polynomial algorithm for the identification of three types of tolerance interval eigenvectors. Bai $Z[5]$ triggers the dynamic of the timing event graph by analyzing the maximal additive algebras, and finally builds the class of timing discrete events.

Through the study of the above scholars, we can conclude that max-plus algebra can be used in many aspects, such as resource allocation, railway system scheduling, optimal control of production lines, image processing and so on. However, there is a lack of analysis of system failure in the above studies, because all the events have the possibility of accidents, so the study of system failure is also a necessary protective measure. Therefore, this paper studies the high speed railway adjustment model of strategy optimization and failure analysis based on max-plus algebra The adjustment and optimization of the train delay are carried out, and at the same time the failure condition is analyzed to eliminate the risk.

\section{MAX-PLUS ALGEBRA}

Definition 1. In the max-plus algebra, the main operations are 
maximization and addition, Defined as follows

$a \oplus b=\max (a, b)$

$a \otimes b=a+b$

for $a, b \in R \cup\{\varepsilon\}$, where $\varepsilon=-\infty$. The structure $\left(R_{\varepsilon}, \oplus, \otimes\right)$ is called the max-plus algebra, denoted by $R_{\max }$, Note that the zero element of the max-plus addition is $\varepsilon=-\infty$, and the identity element of the max-plus multiplication is $e=0$.

The max-plus algebra also has some properties

The commutative law for addition is

$\forall a, b \in R_{\text {max }}, a \oplus b=b \oplus a$

The associative law of addition is

$\forall a, b, c \in R_{\max },(a \oplus b) \oplus c=a \oplus(b \oplus c)$

The commutative law of multiplication is

$\forall a, b, c \in R_{\max },(a \otimes b) \otimes c=a \otimes(b \otimes c)$

The distributive law is

$$
\forall a, b, c \in R_{\max },(a \oplus b) \otimes c=(a \otimes c) \oplus(b \otimes c)
$$

\section{OPTIMIZATION STRATEGY OF HIGH-SPEED RAILWAY TRAIN RUNNING ADJUSTMENT MODEL}

High-speed trains generally do not late at run-time, in most case, it can run normally, but when there is a poor weather conditions, train parts equipment, an accident or emergency, this leads to the delay of the train running and the traffic jam. So when a sudden accident occurs, we need to adjust the time or route of the train. Generally, there are two ways to adjust the sudden accidents of high-speed trains. One is to adopt the method of going along late, and the other is to adopt the method of overtaking [6] (two). The adjustment of the train and the role of the dispatcher's actions depend only on the state of the current situation and the measures taken by the dispatcher, and there is no direct link to the situation before. The adjustment is similar to the characteristics of Markov decision making, so the Markov decision process model can be established[7].

$$
\left\{T, S, A\left(\xi_{e}\right), P\left(\bullet / \xi_{e}, a\right), r\left(\xi_{e}, a\right)\right\}
$$

$T$ is the set of all decision points. $S$ is the set of common conflict states of trains in high-speed railway. $A$ is the set of all actions. $P$ is probability distribution. $r$ is the payment.

\section{A. Two Adjustment Measures of High Speed Train under Sudden Accident}

After the train is delayed, we should consider it according to the time, status and other factors of the train. Generally, two measures will be taken: one is to adopt the postponing trains, and the other is overtaking trains.
The three states of postponing trains are follows. When the state is two trains with the same speed, the front train $i$ is late, while the train $j$ in the rear of the train $i$ is not late, and the delayed time of train $i$ at the station $k+1$ is $t_{w}$, for the rear train $j$, you can adjust the distance between the two trains, It can be reached as soon as possible without traffic accident, and its adjustment time is $t_{x}$. Since the adjustment can be adjusted by adjusting the station's dwell time at the station and the running time of the interval, to restore its normal pass [8], so we can take the postponing trains strategy, as shown in Figure 1 .

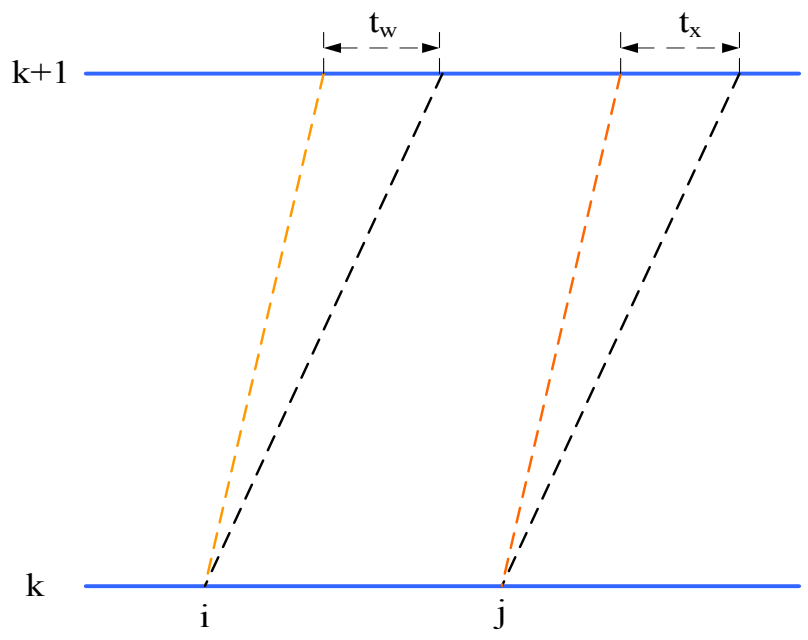

Fig. 1 Adjustment of train running at same speed

When the state is two different speed trains, the front train is a high-speed train, the rear train is medium speed train, the delay time of the front train $i$ at station $k+1$ is $t_{w}$, and the time between the expected arrival time of the train $i$ and the actual arrival time of the rear train $j$ is $t_{x}$. Assume that the minimum time interval is $t_{m}$ between two different trains arriving at the same station. When the $t_{x}>t_{m}$, the rear train does not need to be adjusted. When $t_{x}<t_{m}$, it should be adjusted according to the minimum time interval time. Therefore, we can also take the postponing trains strategy, as shown in Figure 2. 


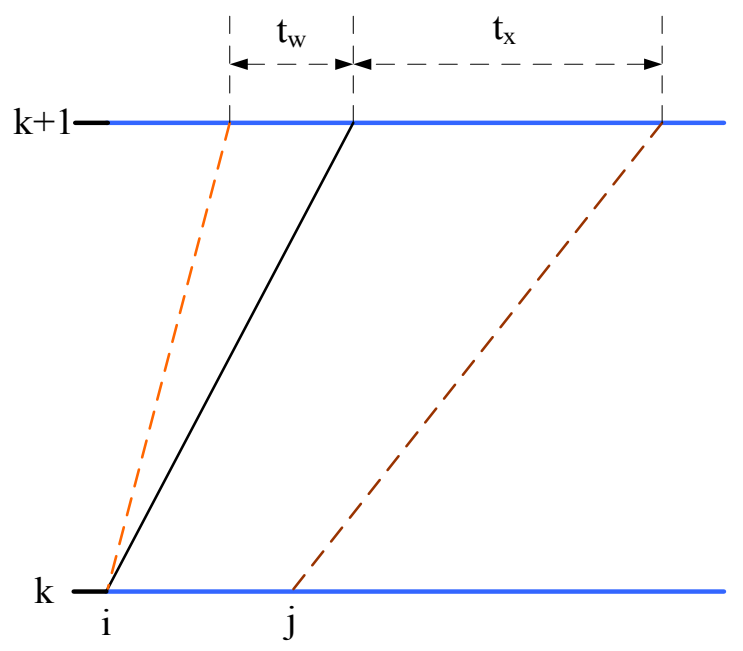

Fig. 2 Adjustment of high and medium speed train running

When the state is Two different speed trains, the front train is a medium speed train, and the rear train is a high-speed train. The front train $i$ is delayed at station $k+1$, so it causes a conflict with the rear train $j$ in the driving area. In this case, the rear train can only be adjusted by the postponing trains strategy, and this adjustment will severely restricts the speed of the rear train $j$, and the effect will be continue in the next driving region, but if the later time does not exceed the maximum acceptable time, and the rear train is also late, the method will make the value of $t_{x}$ is more and more small, so we can also take the postponing trains strategy, as shown in Figure 3.

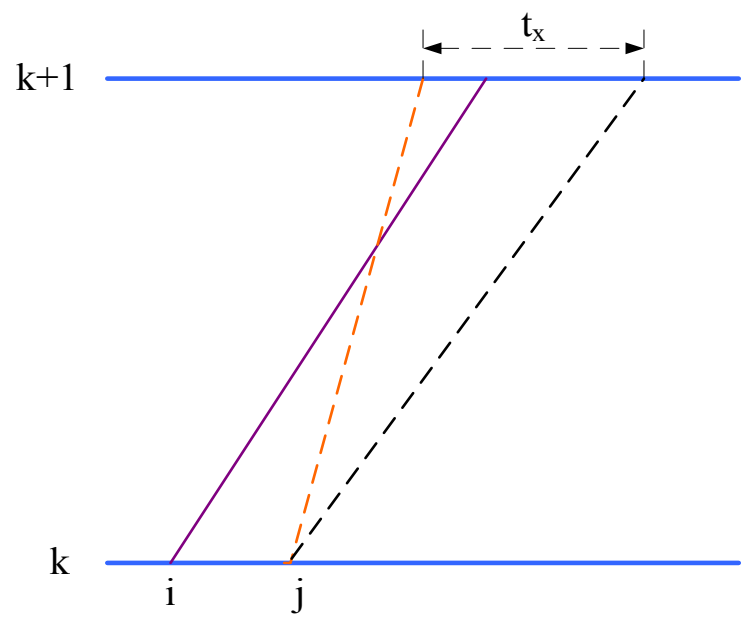

Fig. 3 Adjustment of medium and high speed train running

When the train appeared that serious delay exceeds the maximum time and the rear vehicle does not appear late or rarely occurs, we will no longer use the postponing trains strategy. At this time we need to consider the rear train overtaking the front train. There are two kinds of situations to consider, one is to overtake at the station $K$, the other is to overtake at the station $K+1$, At which station, depending on the train's reward, the higher reward is the optimal adjustment. At the same time, the minimum interval time for the departure of the train is Idd, $t_{0}$ is the time difference between the expected arrival time of the rear train and the front train at station $i$, it is divided into two situations.

When $t_{0}<I d d$, the train overtaking action at station $K$ and station $K+1$ as shown in Figure 4, it can be seen from the figure overtaking action at station $K$ and station $K+1$ can solve the conflict, then we need to calculate the reward $T_{1}, T_{2}$ of the two kinds of the behavior, that is, the weighted total time of train $i$ and train $j$ in the interval,

$$
\begin{aligned}
& T_{1}=l_{j}\left(t_{j}+I_{d d}-t_{0}\right)+l_{i}\left(I_{d d}+I_{f f}+t_{i}\right) \\
& T_{2}=l_{j}\left(t_{i}+I_{d d}-t_{0}\right)+l_{i}\left(t_{i}+I_{d d}+I_{f f}\right)
\end{aligned}
$$

When $t_{0}>I d d$, the train overtaking action at station $K$ and station $K+1$ as shown in Figure 5, it can be seen from the figure, in this case, if the rear train overtaking action at station $K$, we only need to adjust the train $i$, without the need to adjust the train $j$, and then calculate the $T_{1}, T_{2}$ in the case,

$$
\begin{gathered}
T_{1}=l_{j} t_{j}+l_{i}\left(t_{0}+I_{f f}+t_{i}\right) \\
T_{2}=l_{j}\left(t_{i}+I_{d d}-t_{0}\right)+l_{i}\left(t_{i}+I_{d d}+I_{f f}\right)
\end{gathered}
$$

Let $T_{1} \leq T_{2}$, we can get $t_{0} \leq \frac{l_{j}}{l_{i}+l_{j}}\left(t_{i}-t_{j}\right)+I_{d d}$

Let $T_{1}>T_{2}$, we can get $t_{0}>\frac{l_{j}}{l_{i}+l_{j}}\left(t_{i}-t_{j}\right)+I_{d d}$

When $t_{0} \leq \frac{l_{j}}{l_{i}+l_{j}}\left(t_{i}-t_{j}\right)+I_{d d}, T_{1} \leq T_{2}$, if the rear train $j$ overtaking action at station $K$, the weighted total time is small, so the train should $j$ overtaking action at station $K$. When $t_{0}>\frac{l_{j}}{l_{i}+l_{j}}\left(t_{i}-t_{j}\right)+I_{d d}, T_{1}>T_{2}$, that is if the rear train $j$ overtaking action at station $K+1$, the weighted total time is small, so the train should $j$ overtaking action at station $K+1$.

From the above we can see that in the process of high-speed railway train operation adjustment, different situations require different treatment, in general the conflict between the two trains are solved by adjusting the speed of trains and routes, 
ultimately the train operation is optimized without affecting the operation of the train.

\section{B. The Strategy Optimization based on Max Plus Algebra}

Suppose there are $K$ stations and $N$ trains in a section within the period of adjustment, and the following matrices are defined.

The overtaking matrix $O=\left(o_{i, k}\right)_{N \times(K-1)}$, and the matrix element $O_{i, k}$ is the data in line $i$, column $k$, and take the train number of the ith position in the time sequence of the kth operation interval, $i \in\{1, \ldots, N\}, k \in\{1, \ldots, K-1\}$. As shown in Figure 6, matrix the first column data 1,2,3 of the overtaking matrix corresponding to train number 1,2,3in the first operating interval according to the time sequence. The second column data 2,1,3 corresponding to the train number 2,1,3 in second operating interval according to the time sequence. Thus, the position of the train 1 and the train 2 is changed in order. This is because the train 1 at the station $K=2$ is overtaked by the train 2. In turn, the train overtakin matrix in the whole section can be obtained.

The arrival and departure matrix $X=\left(x_{i, k}\right)_{N \times(2 k-2)}$ of the train $i$ at the station $K, x_{i, 2 k-1}$ is the arrival time of the train $i$ at the station $K, x_{i, 2 k}$ is the departure time of the train $i$ at the station $K, k \in\{1, \ldots, K-1\}$. The time delay in the propagation of partial failure, $t_{1}$ to $t_{4}$ as shown in Figure 4.

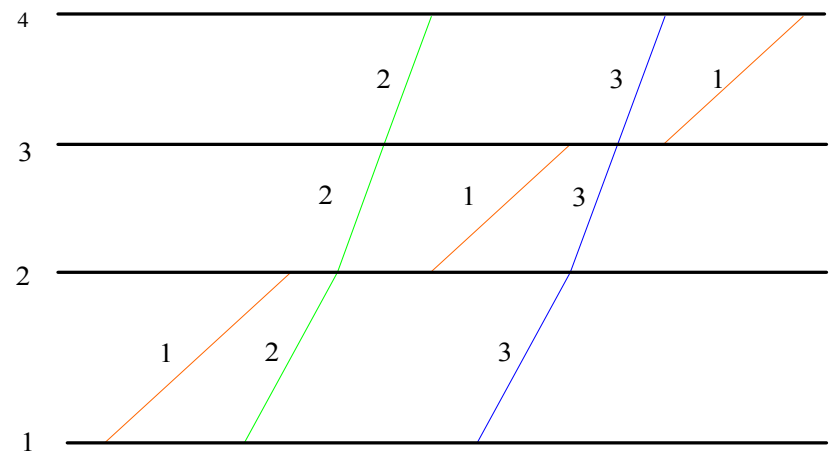

Fig. 4 The train runs between different stations

The corresponding matrix is $\left(\begin{array}{lll}1 & 2 & 2 \\ 2 & 1 & 3 \\ 3 & 3 & 1\end{array}\right)$

The minimum stopping time matrix $t^{\text {stop }}=\left(t_{k}^{\text {stop } i}\right)_{N \times K}$ of the train $i$ at the station $K, t_{k}^{\text {stop,i }}$ is the minimum stop time of the train $i$ at the station $K, i \in\{1, \ldots, N\}, k \in\{1, \ldots, K-1\}$.
The standard running time matrix $t^{r u n}=\left(t_{k}^{r u n, i}\right)_{N \times(K-1)}$ including the additional time of stops between station $K$ and station $K+1, t_{k}^{r u n, i}$ is the standard running time of the train between station $K$ and station $K+1, i \in\{1, \ldots, N\}$, $k \in\{1, \ldots, K-1\}$.

Based on the maximal plus algebra and matrix operations which are given above, we can quickly calculate the train departure time by the state $\xi_{e}$ to the state $\xi_{e+1}$ of all trains in different station under the condition of determinate overtaking sequence and non-conflict train [9].

$x_{1,2 k}$ and $x_{1,2 k+1}$ is the arrival time and the departure time of train 1 at station $K$, and $x_{i, 1}$ is the departure time of each train at the departure station, They are calculated by the following formula.

$$
\left\{\begin{array}{c}
x_{1,1}=0 \\
x_{1,2 k}=x_{1,2 k-1}+t_{k}^{r u n, 1} \\
x_{1,2 k+1}=x_{1,2 k}+t_{k+1}^{s t o p, 1}
\end{array}\right.
$$

$k=2, \ldots, K ; x_{i, 1}=I_{f f}(i-1) ; i=2, \ldots, N$

The calculation formulas of the arrival time $x_{o_{i, k}, 2 k}$ and the departure time $x_{o_{i, k+1}, 2 k+1}$ of the subsequent train at station $K$ are respectively as follows

$$
\begin{gathered}
x_{o_{i, k}, 2 k}=\max \left(x_{o_{i, k}, 2 k-1}+t_{k}^{r u n, o_{i, k}}, x_{o_{i-1, k}, 2 k}+I_{d d}\right) \\
k=1,2, \ldots, K-2 ; i=2, \ldots, N \\
x_{o_{i, k+1}, 2 k+1}=\max \left(x_{o_{i, k}, 2 k}+t_{k+1}^{s t o p, o_{i, k+1}}, x_{o_{i-1, k+1}, 2 k+1}+I_{f f}\right) \\
k=1,2, \ldots, K-2 ; i=2, \ldots, N
\end{gathered}
$$

The formula for the arrival time ${ }^{x_{i, K-1}, 2 k-2}$ of the follow-up train at the final station $K$ is

$$
x_{o_{i, K-1}, 2 K-2}=\max \left(x_{o_{i, K-1}, 2 K-3}+t_{K-1}^{r u n, o_{i, K-1}}, x_{o_{i-1, K-1}, 2 K-2}+I_{d d}\right)
$$

$i=2, \ldots, N$

$y_{i, k}$ is the scheduled arrival time of train $i$ at the final station $K$, formula of the total weighted delay time of all trains is defined as the follows.

$$
t_{\text {all }}=\sum_{i=1}^{N} l_{i}\left(x_{i, 2 K-2}-y_{i, K}\right)
$$




\section{STEADY STATE CHARACTERISTICS OF THE SYSTEM}

Considering the convenience of passengers to use urban rail transit trains and the actual absorption effect of stations for nonuniform distribution of passenger flow, the uniform level of departure and arrival interval is improved to avoid too small station trains from the perspective of the average effective travel time of passenger flow. It is an important objective to improve the operation service level that the headway between departure and arrival results in overload of delayed trains and under-load of subsequent trains, for example, the number of passengers who can be absorbed by subsequent trains is lower than expected due to the short collection time of subsequent passenger flow. Therefore, it is of great significance to study the uniform distribution of station tracking interval between trains in urban rail transit system.

The stability of system state evolution process refers to the boundedness of the evolution process and the convergence of the finite beat of its equilibrium state. It is shown that the boundedness of the evolution process can always be guaranteed, so the stability problem comes down to whether the evolution process can converge to its equilibrium state in a finite number of beats. The equilibrium state is a periodic process, which is usually called periodic steady state. The mathematical definition of stability is given as follows.

The linear system on maximal algebra [10] is

$$
\begin{aligned}
& X(k)=X(k-1) A \oplus U(k) B \\
& Y(k)=X(k) C
\end{aligned}
$$

Where $A(k)$ and $B(k)$ are coefficient matrices, and $C(k)$ is constant which does not change with $k . X(k)$ is state vector, $Y(k)$ is output vector and $U(k)$ is input vector.

For any initial value

$$
X_{0}=\left[x_{01}, \ldots, x_{0 n}\right]^{T}, x_{0 i} \neq \varepsilon, i \in(1, \ldots, n)
$$

if $\lim _{k \rightarrow \infty}\left[x_{i}[k]\right]^{1 / k}=\lambda_{i}$ and the square root is in the sense of maximal algebra, then the system (11) is said to be stable with

the component $x_{i}$. if it is stable for all components, then it is determined that the system is weakly stable.. If $\lambda_{1}=\lambda_{2}=\ldots=\lambda_{n}=\lambda, \lambda$ is a constant, it is determined that the system (11) is stable.

Under the periodic input control, the state quantity of the system must enter the periodic steady state with period one after a limited transition batch. When the system is in the equilibrium state, each station or section is occupied by a train at an average interval of $\lambda$ or $\mu$ for all stations or sections, which depends on the departure interval of the train and the maximum value in the time matrix $T$, which is the tracking interval between trains. Therefore, uniform departure can ensure the uniform distribution of arrival departure interval of trains in the station, and the arrival departure interval of train depends on the departure interval of train and the maximum value of time matrix $T$.

\section{ANALYSIS OF FAILURE PROPAGATION PROCESS UNDER MAX-PLUS ALGEBRA}

The optimization strategy of high speed train adjustment model is studied, however, it could not have toward the best direction for a special case, so the study of failure propagation process will make us nderstand the situation in advance, so as to make accurate judgments in emergencies. Therefore, this paper will study the failure propagation process under maxplus algebra.

The failure propagation process consists of two aspects. The logical chain relation of failure propagation is that the description of the failure system $\mathrm{A}$ includes the other failures $\mathrm{A} 1$ and $\mathrm{A} 2$ that directly cause the system A to occur, and the subsequent failures $\mathrm{A} 3$ and $\mathrm{A} 4$ caused by the system $\mathrm{A}$, as shown in Figure 5.

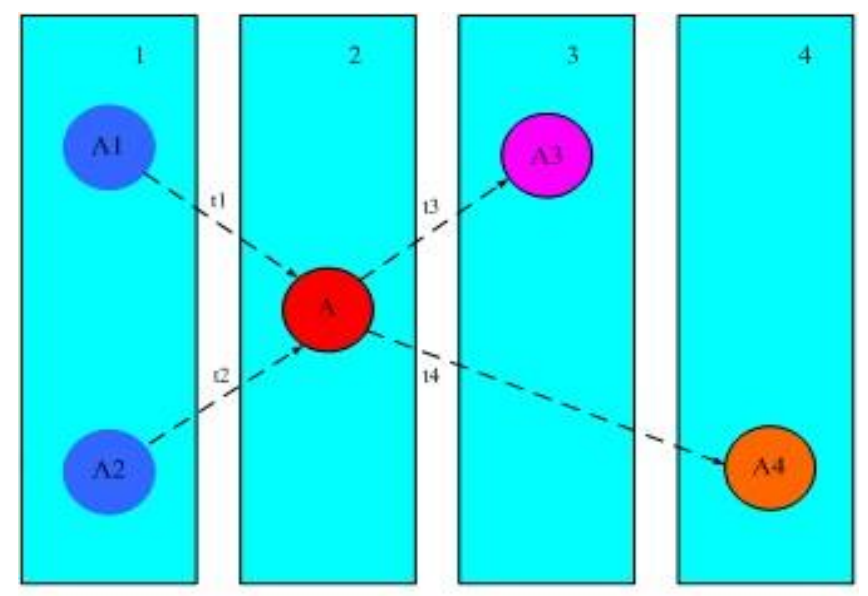

Fig. 5 Logical chain relation of failure propagation

Definition 1: failure propagation matrix is $N \times N$ matrix. $N$ is the type of local failure system in the system. if the ith kind of failure occurs, and after delay $t_{i j}$, the $j t h$ kind of failure occurs, then $\Phi_{s}(i, j)=t_{i j}$, if the $i t h$ kind of failure occurs, it will not affect the occurrence of the $j$ th kind of failure, then $\Phi_{s}(i, j)=\varepsilon, \varepsilon \triangleq \infty$ is the additive zero element in the maxplus algebraic law.

Thus, the failure propagation process is a logic of "or", that is, any failure of the previous stage can result in the occurrence of the latter failure. The iterative calculation should take the minimum cumulative time of the multiple propagation paths. iterative calculation should be minimum of multiple 
propagation paths on the accumulative time. Therefore, the min-plus rule is used to describe the propagation process of failure: an initial state is started, and the final failure propagation state is iteratively obtained by the maxi-plus algorithm. The system state is represented by a $N$ dimension vector

$$
X=\left[X_{1}, X_{2}, \ldots, X_{N}\right]^{T}
$$

where $X_{i}$ indicates the initial time of the $i t h$ kind of failure, let $X(i)=x_{i}$, if the failure has not occurred, then $X(i)=\infty$. the time of occurrence of the failure is unknown $X_{0}$ represents the initial state of the failure, $X_{k}$ indicates the state after the $k$ iterations, $X$ is the final state.The rule of matrix multiplication in max-plus algebra is used to express the change of the system state of failure through one pass.

$$
X_{k+1}=\Phi_{S} X_{k}
$$

The system state changing process of failure which has been passed over many times can be expressed by the power operation of the max-plusl algebra.

$$
\Phi_{S}^{k}=\Phi_{S} \otimes \Phi_{S} \otimes \ldots \otimes \Phi_{S}
$$

The final state of the system is the superimposed of multiple transfer process effects. [22]. $X$ is the final state

$$
\left\{\begin{array}{l}
\Phi_{S}^{*}=E \oplus \Phi_{S} \oplus \Phi_{S}^{2} \oplus \cdots \oplus \Phi_{S}^{N-1} \\
X=\Phi_{S}^{*} X_{0}
\end{array}\right.
$$

Among them, $E$ is the unit matrix in the sense of max-plus algebra, its diagonal element is multiplication zero element, and the rest elements are $\varepsilon$.

For the system of Fig. 2, the failure propagation matrix is

$$
\Phi_{S}=\left|\begin{array}{lllll}
\varepsilon & \varepsilon & \varepsilon & \varepsilon & \varepsilon \\
\varepsilon & \varepsilon & \varepsilon & \varepsilon & \varepsilon \\
t_{1} & t_{2} & \varepsilon & \varepsilon & \varepsilon \\
\varepsilon & \varepsilon & t_{3} & \varepsilon & \varepsilon \\
\varepsilon & \varepsilon & t_{4} & \varepsilon & \varepsilon
\end{array}\right|
$$

It is assumed that the initial state of the system is that Aloccurs at the time $t_{0}$, i.e.:

$$
X_{0}=\left[t_{0}, \infty, \infty, \infty, \infty\right]^{T}
$$

According to equation (54), the final failure status is obtained

$$
\begin{aligned}
X & =X_{0} \oplus X_{1} \oplus X_{2} \\
& =\left[t_{0}, \infty, t_{0}+t_{1}, t_{0}+t_{1}+t_{3}, t_{0}+t_{1}+t_{4}\right]^{T}
\end{aligned}
$$

\section{A. The Failure Control Process based on Maximum Algebra}

This example introduces the max-plus algebra to describe the failure process in the system. Similar to the failure propagation process, the failure control process also includes two aspects: logical chain relation and time delay. Define a similar matrix $\Phi_{E}$ to describe these two attributes. $Y_{0}$ represents the initial state of the failure control process, $Y_{0}$ is set based on the final state of the failure propagation process.

If $X(i)=\infty$ (indicating failure has never occurred), then $Y_{0}(i)=-\infty$ (indicating that the failure does not exist).

If $X(i)=T$ (the failure occurs at time $T$ ), then $Y_{0}(i)=\infty$ (indicates that the failure exists at the initial state and vanishing moments are uncertain).

If the initial value is set that the failure $i$ disappears at the moment $\tau_{0}$, then $Y_{0}(i)=\tau_{0}$. After determining, $Y_{0}$, the final state $Y$ of failure control can be obtained by the following cyclic iteration

$$
\begin{gathered}
Y_{k+1}^{\prime}=\Phi_{E} Y_{k} \\
Y_{k+1}=\min \left\{\left|Y_{k+1}^{\prime}\right|,\left|Y_{k}\right|\right\}
\end{gathered}
$$

Among them, formula (17) is the standard max-plus iterative operation, and the Min operation in formula (18) is to deal with the influence of the special state in the iterative process. The control process of the failure is "and" logical process, only when a failure of the front stage all disappeared, the failure will disappear, iterative calculation should be to maximize the total time of multiple control path, so we use the max-plus algebra rule to describe the failure propagation, when the elements $\pm \infty$ are generated in an iterative operation, if the previous state is a determined value, the subsequent state should hold the value.

For example systems shown in Figure 6, the failure control matrix is set as

$$
\Phi_{E}=\left[\begin{array}{lllll}
\varepsilon & \varepsilon & \varepsilon & \varepsilon & \varepsilon \\
\varepsilon & \varepsilon & \varepsilon & \varepsilon & \varepsilon \\
\tau_{1} & \tau_{2} & \varepsilon & \varepsilon & \varepsilon \\
\varepsilon & \varepsilon & \tau_{3} & \varepsilon & \varepsilon \\
\varepsilon & \varepsilon & \varepsilon & \varepsilon & \varepsilon
\end{array} \mid\right.
$$

Note that $\Phi_{S}$ and $\Phi_{E}$ are asymmetric

$$
\begin{aligned}
& \Phi_{S}(5,3)=t_{4} \\
& \Phi_{E}(5,3)=\varepsilon
\end{aligned}
$$

Formula (13), (14) indicate that A can trigger A4, but A disappears without causing the A3 to disappear. This is an important characteristic of failure in the propagation process. 
The method presented in this paper can be used to analyze this kind of characteristics.

Assuming that the A1 vanishes at time $\tau_{0}$, the initial state of the failure control process is

$$
Y_{0}=\left[\tau_{0},-\infty, \infty, \infty, \infty\right]^{T}
$$

By using (52) and (53) iterations, the final state of the failure control process can be obtained

$$
Y=\left[\tau_{0},-\infty, \tau_{0}+\tau_{1}, \tau_{0}+\tau_{1}+\tau_{2}, \infty\right]^{T}
$$

\section{B. Failure Analysis Method based on Max-plus Algebra}

The steps of failure analysis based on max-plus algebra are as follows.

Step1. According to the system security function, determine the system hazards that need to be analyzed, that is, the dangerous failure of the system boundary.

Step2. According to the method proposed above, the failure exposure time is calculated, and the system hazards are controlled by (23).

$$
T_{s f}(h)=Y(h)-X(h)<P S T_{h}
$$

Among them, $h$ is the system boundary risk failure index number that is to be analyzed, $P S T_{h}$ indicates the process safety time of the failure process, that is, tolerable dangerous output duration. $X(h)$ is obtained by formula (21), $Y(h)$ is obtained by formula (22) and (23).

Make an explanation of (23) from two aspects. If no harm occurs, then $Y(k)=-\infty, X(k)=\infty, Y(k)-X(k)=-\infty<P S T_{h}$, the content is satisfied (23).

If a hazard occurs, $X(k)$ is the delay time when the hazard occurs, $Y(k)$ indicates the delay time when the hazard is controlled, and meeting (23) represents risk within the system acceptable range, otherwise, system risk will not be accepted.

Through the analysis of the failure of the train we can draw: This method can analyze the failure propagation mechanism in the whole system in the case of multi-module collaboration system with complex interaction, the quantitative failure propagation and control analysis are carried out to effectively identify the system risks and provide relevant functions and parameter constraints for subsequent engineering design.

\section{CONCLUSION}

In this paper, according to the max-plus algebra method, the adjustment model of high-speed railway train is optimized. The train adjustment mode in different cases is analyzed, and the running time matrix is established., calculate the arrival and departing time of all trains at different stations, and then get the optimal adjustment method.. Then, taking into account all the trains have emergency, we analyzed the failure and dissemination under max-plus algebra sense, thus The quantitative failure propagation and control analysis can be carried out to identify the risk of the system.

After all, train operation adjustment is a large and complex subject with many influencing factors and strong applicability. For the actual technical system of train operation command automation system, the work done in this paper can only be regarded as the most basic research, and there are still many problems to be discussed in depth. Moreover, with the development of high-speed railway from the research stage to the planning and design stage, some preconditions are constantly changing, so the research on train operation adjustment must be deepened and expanded accordingly. In this paper, the train operation process is divided into several arrival and departure events to deal with, as for the train operation in the section is not considered in detail. With the determination of high-speed railway signal system and line direction, it is necessary to further improve the research on train operation organization in the section. Train operation adjustment is a work with high intelligent requirements. Although some intelligent methods are introduced in this paper, it is not enough from the practical level. It is necessary to further improve the intelligent level to reduce the man-machine dialogue operation, so as to improve the automation degree of train operation adjustment and better meet the timeliness requirements of high-speed railway train operation adjustment.

\section{REFERENCES}

[1] Fard H M, Prodan R, Fahringer T. "A Truthful Dynamic Workflow Scheduling Mechanism for Commercial Multicloud Environments". IEEE Transactions on Parallel \& Distributed Systems, 2019, 24(6), pp. 1203-1212.

[2] Chen Jin, Liu Shuhong. "Modeling method of minimax algebra based on the method of Job-Shop production line". Journal of System Simulation, 2018, 326. (02), pp.310-312.

[3] LU Zhi-wei, TANG Xin-min, HAN Song-chen. "Study on collision-free 4D path of multi-aircraft based on maximal algebra". Chinese Journal of Transportation and Safety, 2018, 30 (03), pp.147-151.

[4] Zhou X, Zhang Q, Xu W, et al. Intelligent Adjustment Method for Train Operation Diagram with Consideration of Motor Train Set Connection. Tiedao Xuebao/Journal of the China Railway Society, 2018, 40(8):19-27.

[5] Bai Z, Zhou L, Wang J, et al. The Evaluation Method and Index System for High-Speed Railway Train Operation Plan. Lecture Notes in Electrical Engineering, 2017, 287:519-526.

[6] Mock R, Corvo M. Risk analysis of information systems by event process chains. International Journal of Critical Infrastructures, 2018, 32(2-3), pp. 247-257.

[7] Lszl Bernyi, Relationship between the number of ISO 9001 certifications and the national economic performance in transitional economies, WSEAS Transactions on Business And Economics, pp. 99-112, Volume $15,2018$.

[8] Marek Kubalcik, Vladimir Bobal, Tomas Barot, Statistical Analysis of Modified Predictive Control of Non-Minimum Phase System, WSEAS Transactions on Applied and Theoretical Mechanics, pp. 205-211, Volume 14, 2019.

[9] Munteanu I, Cutululis N A, Bratcu A I, et al. "Optimization of variable speed wind power systems based on a LQG approach". Control Engineering Practice, 2005, 13(7), pp. 903-912.

[10] Chen B, Potts C N, Woeginger G J. "A review of machine scheduling: Complexity, algorithms and approximability", Handbook of combinatorial optimization. Springer US, 1998: 1493-1641.

\section{Creative Commons Attribution License 4.0} (Attribution 4.0 International, CC BY 4.0)

This article is published under the terms of the Creative Commons Attribution License 4.0 https://creativecommons.org/licenses/by/4.0/deed.en_US 